Article

\title{
Investigation of Tunable Surface Plasmon Resonances on Spheroid Core-Shell Alloy Nanoparticles Using DDA Method
}

\author{
Jing Liu 1, Chean-Cheng Su 2, Qiubo Ye 1, Wei Chen 1, Yushan Chen 1,* , Cheng-Fu Yang 2,* \\ 1 School of Information Engineering, Jimei University, Xiamen, Fujian 361021, PR China; jingliu@jmu.edu.cn (J.L.), \\ qbye@jmu.edu.cn(Q.Y.), chenweiniceway@gmail.com (W.C.) \\ 2 Department of Chemical and Material Engineering, National University of Kaohsiung, Kaohsiung 811, Taiwan; \\ ccsu@nuk.edu.tw (C.S.) \\ * Correspondence: chenys@jmu.edu.cn, cfyang@nuk.edu.tw
}

\begin{abstract}
In this work, numerical simulations for the absorption and scattering efficiencies of spheroid core-shell nanoparticles (CSNs) were conducted and studied using the discrete-dipole approximation method. The characteristics of surface plasmon resonances (SPR) depend upon shell thickness, the compositions of the core and shell materials, and the aspect ratio of the constructed CSNs. We used different core@shell compositions, specifically $\mathrm{Au} @ \mathrm{SiO}_{2}, \mathrm{Ag} @ \mathrm{SiO}_{2}, \mathrm{Au} @ \mathrm{TiO}_{2}, \mathrm{Ag} @ \mathrm{TiO}_{2}, \mathrm{Au} @ \mathrm{Ag}$, and $\mathrm{Ag} @ \mathrm{Au}$, for extinction spectra analysis. We also investigated coupled resonance mode wavelengths by adjusting the composition's layer thickness and aspect ratio. In this study, we show that the extinction efficiency of the Ag@ $\mathrm{TiO}_{2}$ core-shell nanoparticles (CSNPs) was higher than that of the others, and we examined the impact of $\mathrm{TiO}_{2}$ shell thickness and $\mathrm{Ag}$ core radius on SPR peak positions. From the extinction spectra we found that the $\mathrm{Ag}_{\mathrm{TiO}}$ nanoparticle had better refractive index sensitivity and figure of merit when the aspect ratio was set to 0.3. All of the experimental results proved that the tunability of these plasmonic resonances was highly dependent on the material used, the layer thickness, and the aspect ratio of the core@shell CSNPs.
\end{abstract}

Keywords: surface plasmon resonance; core-shell nanoparticles; discrete-dipole approximation; aspect ratio

\section{Introduction}

Core-shell nanoparticles (CSNPs) are nanostructured materials with interesting characteristics that offer a broad range of applications in optics, biomedicine, environmental science, materials, catalysis, and energy because they have excellent properties such as versatility, tunability, and stability [1-3]. They have attracted tremendous attention for their dramatically tunable optical properties.

Metal nanoparticles (NPs) have special optical properties because they possess more characteristics and functions than the same materials when they are in bulk structures. Smaller particle size gives rise to increased surface-area-to-volume ratios and strong adsorption capacity in the visible region [4]. One of the extremely impressive optical properties of metal NPs is their surface plasmon resonances (SPR). In this situation, optical properties are enhanced and the tunability of these SPR peaks is highly dependent on structural characteristics such as shell thickness, the composition of the core and shell materials, aspect ratio, and the diameter of the spheroidal NPs, as well as their external dielectric environment. However, it is difficult to determine the real influences of these parameters on the optical properties of NPs when we use SPR in various applications, such as optics, biosensors, and medical diagnostics [5].

NPs in complex, multilayer, or core-shell nanostructures have attracted significant interest because they can offer additional opportunities for innovation with tailored materials in the fields of physics [6], nanomaterials [7], biomedical nanosystems [8], optical chemical sensors [9], magnetic nanocomposites [10], engineering chemistry [11], photocatalysts [12], solar cells [13], and electrical nanosystems [14]. Among these special structures, the core-shell is a particular class of NPs, consisting of a core and one or several shell layers. SPR tunability can be achieved by using different materials to construct the combination of core and shell layers; the diverse applications of CSNPs depend on the core's and shell's properties. Many different categories of CSNPs based on various core and shell materials have been investigated, including CSNPs with metal@metal, metal@dielectric, and dielectric@metal structures [15-17]. The different kinds of CSNPs have attracted plenty of attention, since they have a wide range of applications in 
different fields, such as photovoltaics and optics, biological labeling, catalysis, photonic crystal creation, optical bioimaging, information storage, and pharmaceuticals [18, 19].

Among the noble metals that exhibit SPR, gold and silver are the most commonly investigated, as they have the most exciting selective absorption in the visible range [20] and their SPR characteristics can easily be adjusted via their size and shape. The synthesis of stable plasmonic NPs is very important, because they have unique surface properties and are suitable for a variety of research fields. In addition, the near-field enhancement of Ag NPs is estimated to be greater than 10 times that of similar Au NPs. Ag NPs are therefore especially attractive applications in SPR devices. It is well known that metallic $\mathrm{Ag}$ is easily oxidized into $\mathrm{Ag}_{2} \mathrm{O}$ in air, which has greatly limited its practical application. If a layer of stable oxides is coated on the surface of Ag NPs, the oxidization of Ag NPs can be prevented. Among numerous oxides, $\mathrm{TiO}_{2}$ has been identified as effective because it has high photocatalytic ability, excellent chemical stability, low toxicity, and low cost [21].

To realize the complementary advantages of $\mathrm{Ag}$ and $\mathrm{TiO}_{2} \mathrm{NPs}$, Pastoriza-Santos et al. first synthesized $\mathrm{Ag} @ \mathrm{TiO}_{2}$ CSPs and investigated their properties [22]. They explored a novel method to construct a $\mathrm{Ag} @ \mathrm{TiO}_{2} \mathrm{CSP}$ nanostructured system, composed of silver cores and a 1-2 nm layer of titanium oxide produced by the simultaneous reduction of silver and condensation of titanium butoxide. A few years later, Sherif et al. tried to explain the contribution that the outer $\mathrm{TiO}_{2}$ shell made in enhancing the sensitivity of the Ag core. They applied $\mathrm{TiO}_{2}$, which has a fairly high refractive index of $\sim 2.2$, as an effective dielectric layer to enhance the sensor sensitivity of the lower Ag layer. They also found that the $\mathrm{TiO}_{2}$ layer could be a process-compatible protection layer for preventing the $\mathrm{Ag}$ substrate from oxidizing [23]. However, the effect of the aspect ratio and size of the CSNPs has not been widely discussed.

To understand the optical behavior of CSNPs, such as their sensitivity and quality factors, the discrete-dipole approximations (DDA) technique can be employed to find the effects of shell thickness and core diameter on the SPR properties of fabricated CSNPs. DDA is one of the most popular numerical analysis tools to calculate the optical properties of designed CSNPs, such as the absorption cross-section, scattering cross-section, and extinction (absorption + scattering) cross-section of metallic NPs and CSNPs with arbitrary shapes, sizes, and structures [24]. In this study, we investigate the relationship between the wavelength and the extinction spectra of coated spheroid CSNPs with different core@shell compositions. To examine and realize the tunability of SPR peaks, we simulate CSNPs of various compositions: $\mathrm{Au} @ \mathrm{SiO}_{2}, \mathrm{Ag} @ \mathrm{SiO}_{2}, \mathrm{Au} @ \mathrm{TiO}_{2}, \mathrm{Ag} @ \mathrm{TiO}_{2}$, Au@Ag, and Ag@Au. According to theoretical calculations, we find that the wavelength of the SPR peaks depends on the thickness of the shell layer, the size of the CSNPs, the surrounding environment, and the materials chosen for the core and shell. In addition, we observe the impact of spherical core-shell thickness on the SPR peaks' wavelengths and corresponding spectral widening in distinct regimes of the spectrum. Finally, we explore wavelength variability in the SPR peaks by changing the aspect ratio of the fabricated CSNPs. Through DDA calculations and experimental results, we reach a valuable conclusion.

\section{DDA Method}

The DDA is a convenient method for describing the scattering of light from the surfaces of NPs with arbitrary shapes. In DDA formalism, the object is described as a cubic array lattice of electric dipoles (called N-point dipoles). In the electric dipoles, the polarizability and position vector of each dipole are specified as $\alpha_{i}$ and $r_{i}$, respectively. The induced dipole polarization $P_{\mathrm{i}}$ in each element is determined from:

$$
\boldsymbol{P}_{i}=\alpha_{i} \boldsymbol{E}_{10 c, i}\left(\boldsymbol{r}_{i}\right), i=1,2, \ldots, N
$$

where the local field Eloci $\left(\mathrm{r}_{\mathrm{i}}\right)$ is defined as the sum of the field radiated from all the other N-1 dipoles. For a given special wavelength $\lambda$, including the contribution of all the other dipoles, the local field can be written as

$$
\boldsymbol{E}_{10 c, i}\left(\boldsymbol{r}_{i}\right)=\boldsymbol{E}_{0} \exp \left(i \boldsymbol{k} \cdot \boldsymbol{r}_{i}\right)-\sum_{\substack{j=1 \\ i \neq j}}^{N} \boldsymbol{A}_{i j} \boldsymbol{P}_{j}, i=1,2, \ldots, N
$$

where $\mathrm{k}$ and $\mathrm{E}_{0}$ are defined as the wave vector and the amplitude of the incident radiation. The total generated electric field at position $i$ caused by the dipole generated at position $\mathrm{j}$ is presented in the second term of equation (2), which can normally be expressed in terms of the dipole-dipole interaction in matrix A as: 


$$
\boldsymbol{A}_{i j} \boldsymbol{P}_{j}=\frac{\exp \left(i \boldsymbol{k} r_{i j}\right)}{r_{i j}^{3}}\left\{\boldsymbol{k}^{2} \boldsymbol{r}_{i j} \times\left(\boldsymbol{r}_{i j} \times \boldsymbol{P}_{j}\right)+\frac{1-i \boldsymbol{k} r_{i j}}{r_{i j}^{2}} \times\left[r_{i j}^{2} \boldsymbol{P}_{j}-3 \boldsymbol{r}_{i j}\left(\boldsymbol{r}_{i j} \cdot \boldsymbol{P}_{j}\right)\right]\right\}(j \neq i)
$$

When equations (2) and (3) are substituted into (1), the system equation (4) can be generated:

$$
\boldsymbol{A}^{\prime} \cdot \stackrel{\mathbf{P}}{\boldsymbol{P}}=\stackrel{1}{\boldsymbol{E}}
$$

where the off-diagonal elements of the matrix, $\boldsymbol{A}_{\mathrm{ij}}^{\prime}$ are the same as $\boldsymbol{A}_{\mathrm{ij}}$ for a system with total dipoles, N, whereas, $\stackrel{\boldsymbol{E}}{\boldsymbol{E}}$ and $\stackrel{\boldsymbol{P}}{\boldsymbol{P}}$ are $3 \mathrm{~N}$-dimensional vectors, and $\boldsymbol{A}^{\prime}$ is a $3 \mathrm{~N} \times 3 \mathrm{~N}$ matrix. If we solve this set of $3 \mathrm{~N}$ complex using linear equations, the polarizations $\stackrel{\boldsymbol{P}}{\boldsymbol{P}}$ can be determined. We can then calculate the cross-section of the extinction factor as follows:

$$
C_{e x t}=\frac{4 \pi \boldsymbol{k}}{\left|\boldsymbol{E}_{0}\right|^{2}} \sum_{i=1}^{N} \operatorname{Im}\left(\boldsymbol{E}_{1 o c, i}^{*} \cdot \boldsymbol{P}_{i}\right)
$$

\section{Results and Discussion}

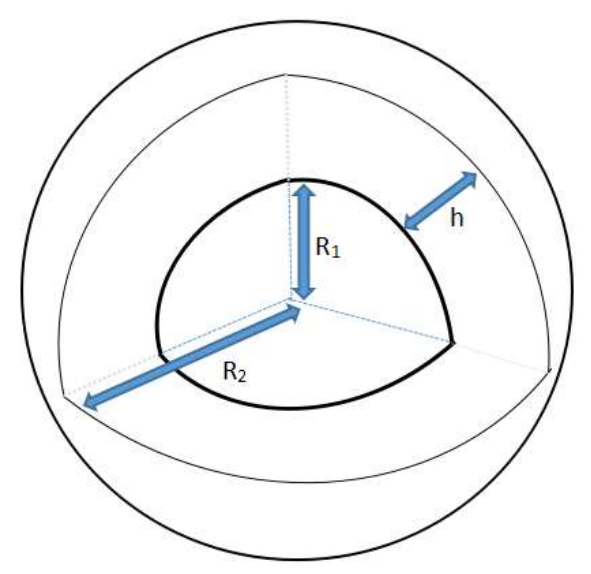

Figure 1. Schematic diagram of a core-shell structure

Figure 1 shows a schematic diagram of the proposed model with different scales in nanometers. The core-shell structured composite NPs can be viewed as having two parts. One is a nucleus wrapped in the interior of the NPs, and the other is a shell that surrounds the nuclear NPs. R1 represents the core radius of the core-shell structure, and $\mathrm{R}_{2}$ represents the radius of the whole core-shell structure, while $\mathrm{h}$ is the shell thickness. To explore the SPR characteristics, we used Ag and $\mathrm{Au}$ as the core layer of the nanospheroid since they have good extinction properties.

In this paper, we investigate three different combinations to achieve spherical CSNPs. The first uses a noble metal ( $\mathrm{Ag}$ and $\mathrm{Au})$ as the core, and the shell layer is coated with a dielectric material of $\mathrm{SiO}_{2}$. The second uses a noble metal ( $\mathrm{Ag}$ and $\mathrm{Au}$ ) as the core, and the shell layer is coated with a dielectric material of $\mathrm{TiO}_{2}$. The last combination is metal@metal with the structures of Ag@Au and Au@Ag. We demonstrate that by altering the effective radii of the NPs and the thickness of the shell layer, we achieve wide tunability in the wavelengths of the SPR peaks. Using the DDA method, the extinction patterns of these various CSNPs with their different combinations are compared with single noble metal nanospheres (Ag and $\mathrm{Au}$ ), and the results are shown in Figure 2. 


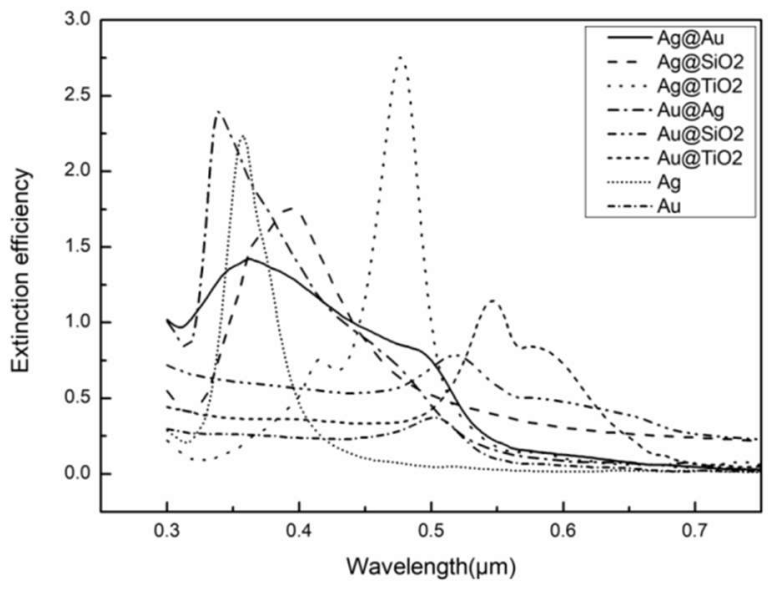

Figure 2. Comparison of simulation results for various core-shell structures

We observe that the wavelengths of the SPR peaks generated by the CSNPs can be tuned for the spectrum from the visible to the near-infrared region by manipulating the core and shell composition. Compared with uncoated nanospheres, the CSNPs provide an extra degree of freedom in the SPR peaks, meaning that the SPR peaks of the electromagnetic spectra can be tuned and shifted to the desired range. There are many advantages to coating a shell layer on the surfaces of uncoated NPs to form CSNPs structures-stability, surface modification, reduction in consumption of precious materials, and reduction in reactivity. The results shown in the extinction spectra suggest that the $\mathrm{Ag} @ \mathrm{TiO}_{2}$ CSNP structure has the highest sensitivity with different materials. Because the Ag@TiO $\mathrm{CSNPs}_{2}$ exhibited the optimum SPR characteristics, we chose them for our follow-up study.

Next, we studied the relationships between the wavelength of an SPR peak and the core radius as well as the shell thickness, since both are important parameters used to tune SPR wavelengths from the ultraviolet to the infrared domain. Figure 3 shows extinction efficiency versus wavelength for a fixed core $\left(R_{1}=25 \mathrm{~nm}\right)$ and various shell thicknesses from 1 to $5 \mathrm{~nm}$, while the corresponding total thickness $\mathrm{R}_{2}$ was changed from 26 to $30 \mathrm{~nm}$.

Figure 3(a) clearly shows that as the shell layer thickness (h) increased from 1 to $5 \mathrm{~nm}$, the wavelength of the SPR peak shifted from 361 to $448 \mathrm{~nm}$. This result suggests that as the shell layer thickness increases, the extinction spectrum exhibits the red-shift phenomenon, and the extinction peak is gradually reduced. The RIS can be defined as $\mathrm{m}=\Delta \lambda / \Delta \mathrm{n}$ [25], where $\Delta \lambda$ and $\Delta \mathrm{n}$ are the variation in the wavelength of the SPR peak and the variation in the refractive index, respectively. For CSNPs with a diameter of $25 \mathrm{~nm}$ for the Ag core and a thickness of $5 \mathrm{~nm}$ for the $\mathrm{TiO}_{2}$ layer, the wavelength of the SPR peak experienced a red shift when the refractive index $\mathrm{n}$ increased, as shown in Figure 3(b). When the refractive index increased from 1.0 to 1.05, the peak wavelength shifted from 449 to $453 \mathrm{~nm}$, exhibiting an RIS of $76 \mathrm{~nm} / \mathrm{RIU}$ (refractive index unit), as indicated in Figure 3(c). Figure 3(e) shows the RIS values of CSNPs with $\mathrm{TiO}_{2}$ shell layers of different thicknesses. Based on the calculated results, we prove that the thickness of the $\mathrm{TiO}_{2}$ layer has a large effect on the RIS value of the $\mathrm{Ag} @ \mathrm{TiO}_{2}$ CSNPs. When the thickness increased from 1 to 5

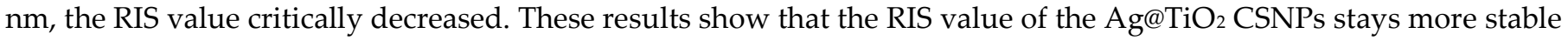
and has a higher value when the thickness of the $\mathrm{TiO}_{2}$ shell layer is $1 \mathrm{~nm}$. The $\mathrm{FOM}$ value for a metal nanostructure is defined as $\mathrm{p}=\mathrm{S} / \mathrm{W}$ [26], where $\mathrm{S}$ and $\mathrm{W}$ denote the RIS value and FWHM value, respectively. Using the results in Figures 3(e) and 3(d), we calculated the FOM values and present the results in Figure 3(f), which shows that the FOM value decreased as the $\mathrm{TiO}_{2}$ layer thickness increased from 1 to $5 \mathrm{~nm}$. The maximum FOM value was obtained when the thickness of the $\mathrm{TiO}_{2}$ layer was set at $1 \mathrm{~nm}$. 


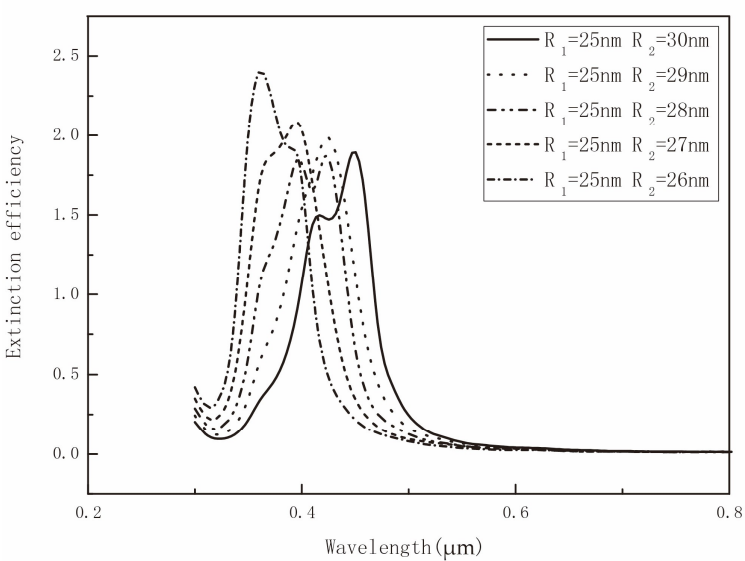

(a)

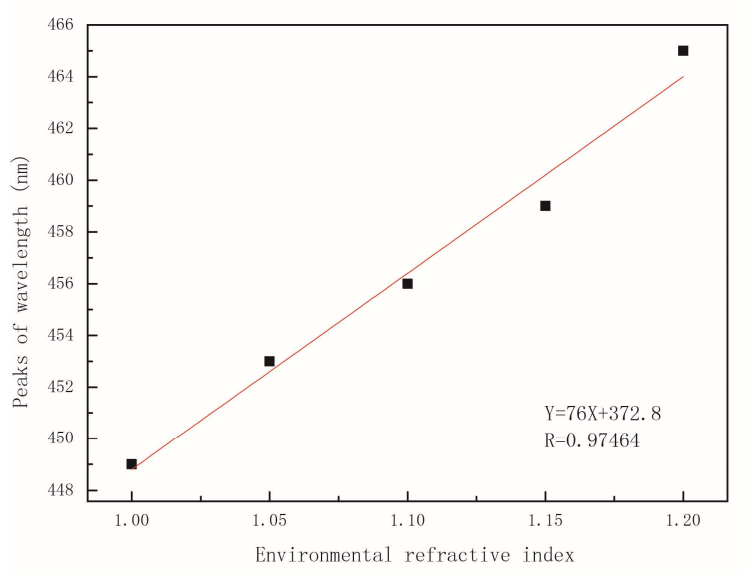

(c)

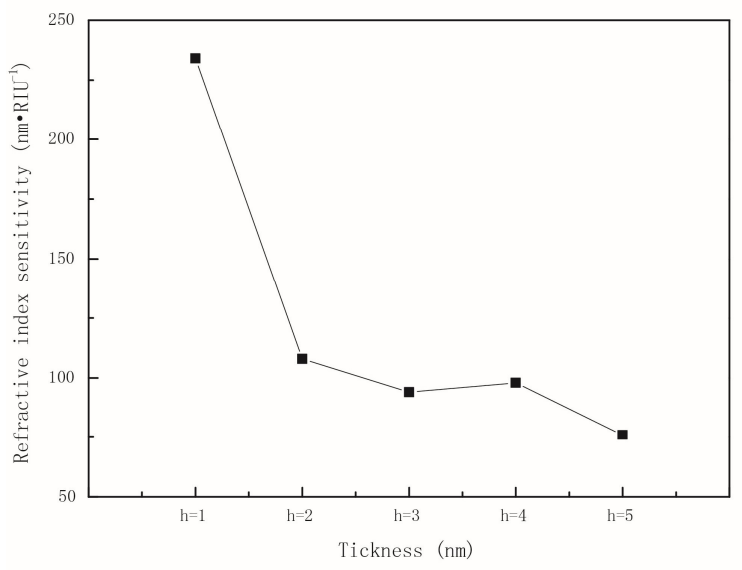

(e)

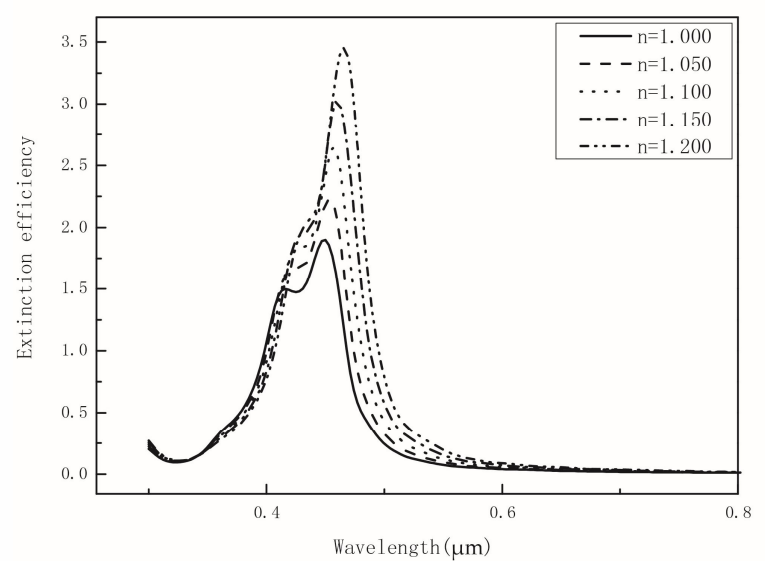

(b)

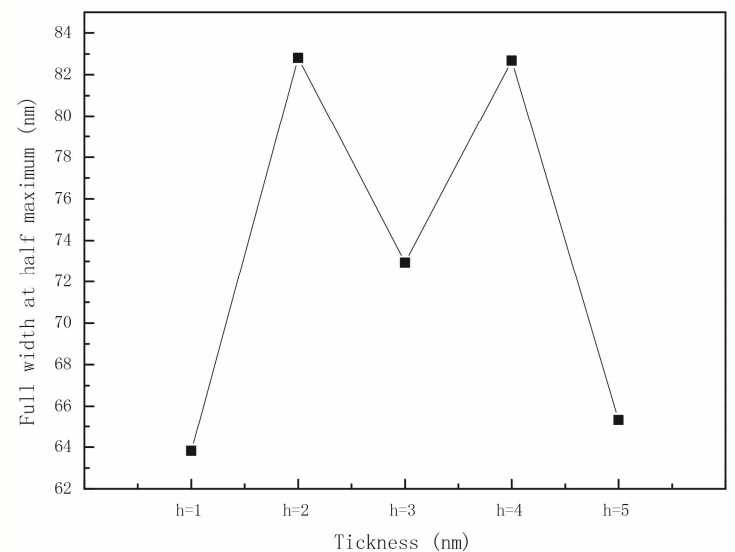

(d)

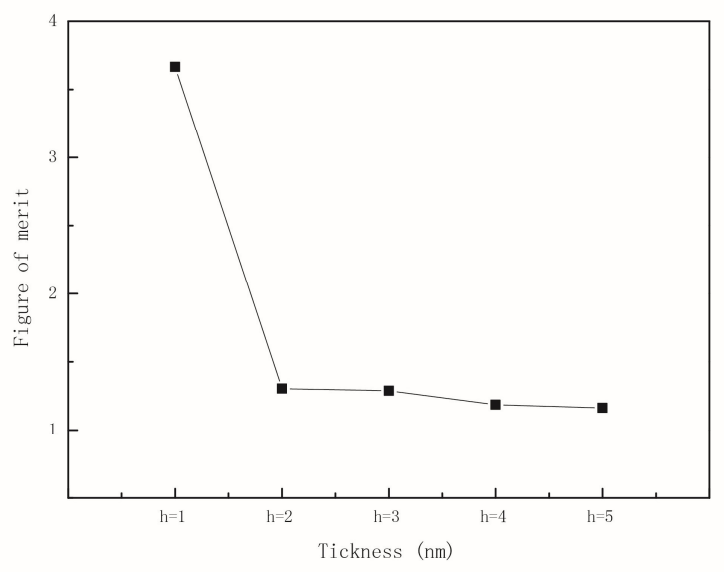

(f)

Figure 3. Calculated results for $\mathrm{Ag} @ \mathrm{TiO}_{2}$ core-shell structures: (a) extinction spectra for a fixed core $\left(\mathrm{R}_{1}=25 \mathrm{~nm}\right)$ with different shell thickness; (b) extinction spectra for a $25 \mathrm{~nm}$ Ag layer and $5 \mathrm{~nm} \mathrm{TiO}_{2}$ layer in different media; (c) refractive index sensitivity curve for a $25 \mathrm{~nm} \mathrm{Ag}$ layer and $5 \mathrm{~nm} \mathrm{TiO}$ layer; (d) full width at half maximum as a function of shell thickness with a fixed core $\left(R_{1}=25 \mathrm{~nm}\right)$; (e) refractive index sensitivity distributions for a fixed core ( $R_{1}$ $=25 \mathrm{~nm}$ ) with different shell thickness; (f) figure of merit for a fixed core with different shell thicknesses. 


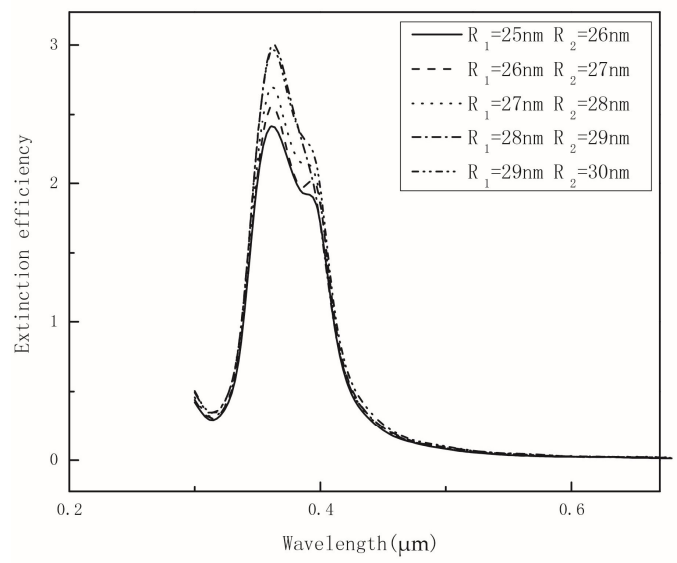

(a)

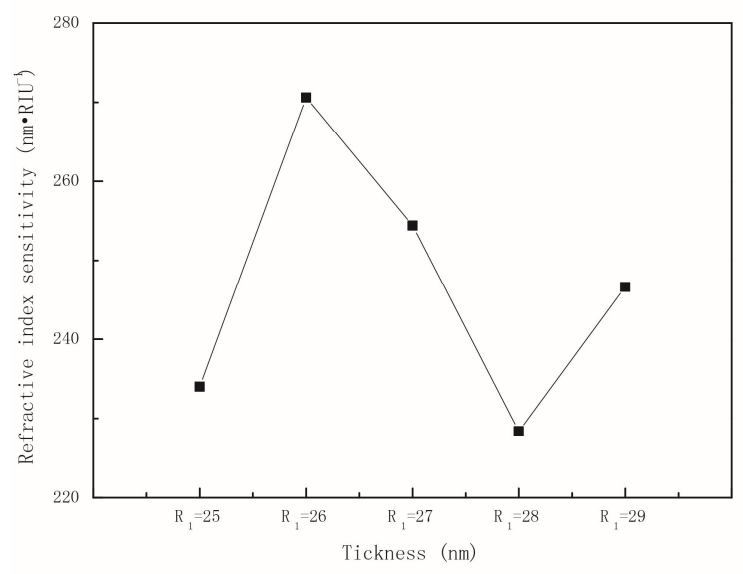

(c)

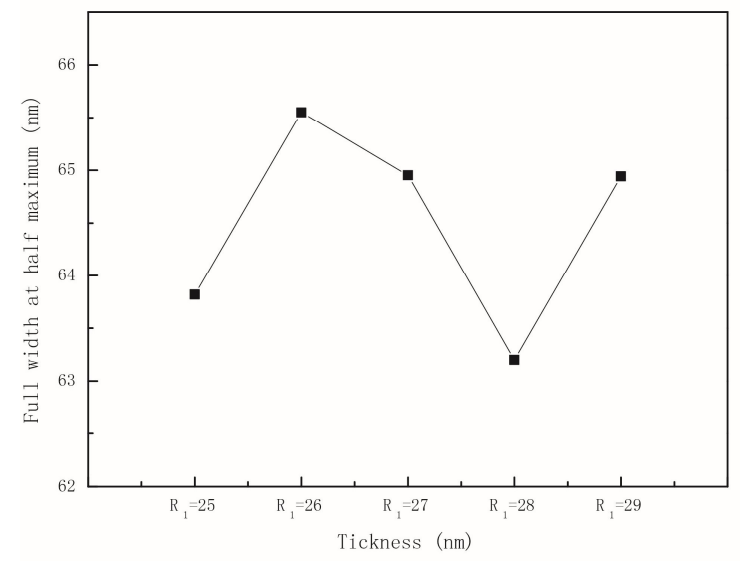

(b)

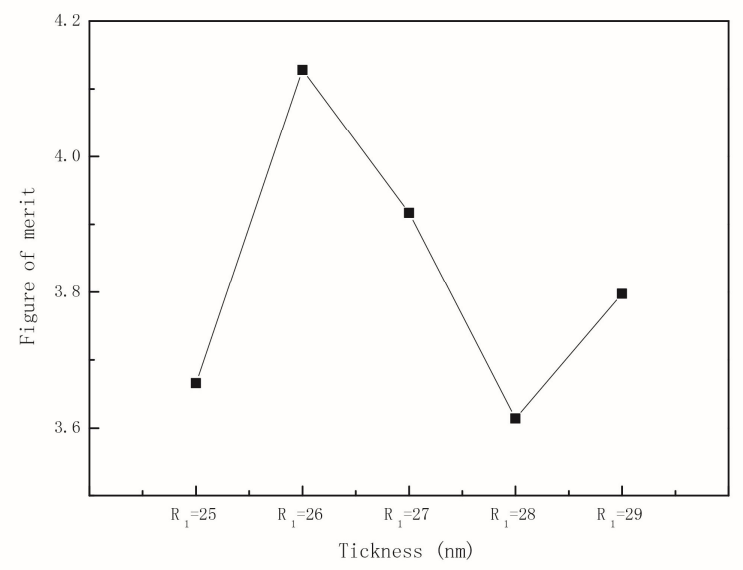

(d)

Figure 4. Calculated results for $\mathrm{Ag} @ \mathrm{TiO}_{2}$ core-shell structures with fixed shell thickness $(\mathrm{h}=1 \mathrm{~nm})$ and different core radii (25-29 nm): (a) extinction spectra; (b) full width at half maximum; (c) refractive index sensitivity distributions; (d) figure of merit.

We conducted further analysis using a fixed shell thickness $(1 \mathrm{~nm})$ and varying the core radius from 25 to $29 \mathrm{~nm}$,

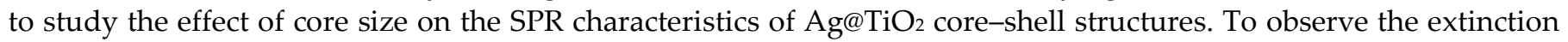
properties of the nucleus as its radius increased, we used the DDA algorithm to calculate the results of the extinction spectra; these are shown in Figure 4(a). Figures 4(c) and 4(d) represent the RIS and FOM of the Ag@TiO $\mathrm{CSNPs}_{\text {with }}$ an Ag layer of different thicknesses and a shell thickness (h) of $1 \mathrm{~nm}$. Based on the simulation results, we observe that the RIS and FOM of the Ag@TiO 2 CSNPs are more stable and have optimum performance and higher values when the Ag layer thickness is $26 \mathrm{~nm}$.

It has been reported that SPR peaks in the extinction spectra of CSNPs can also be tuned from the visible light region to the near-infrared region by manipulating their aspect ratio-that is, by changing the aspect ratio of the entire core-shell structure, as shown in Figure 5. To explore the different aspect ratios in terms of extinction performance, we used an aspect ratio ranging from 0.2 to 1 . Based on the DDA method, the resulting extinction spectra are shown in Figure 6. 


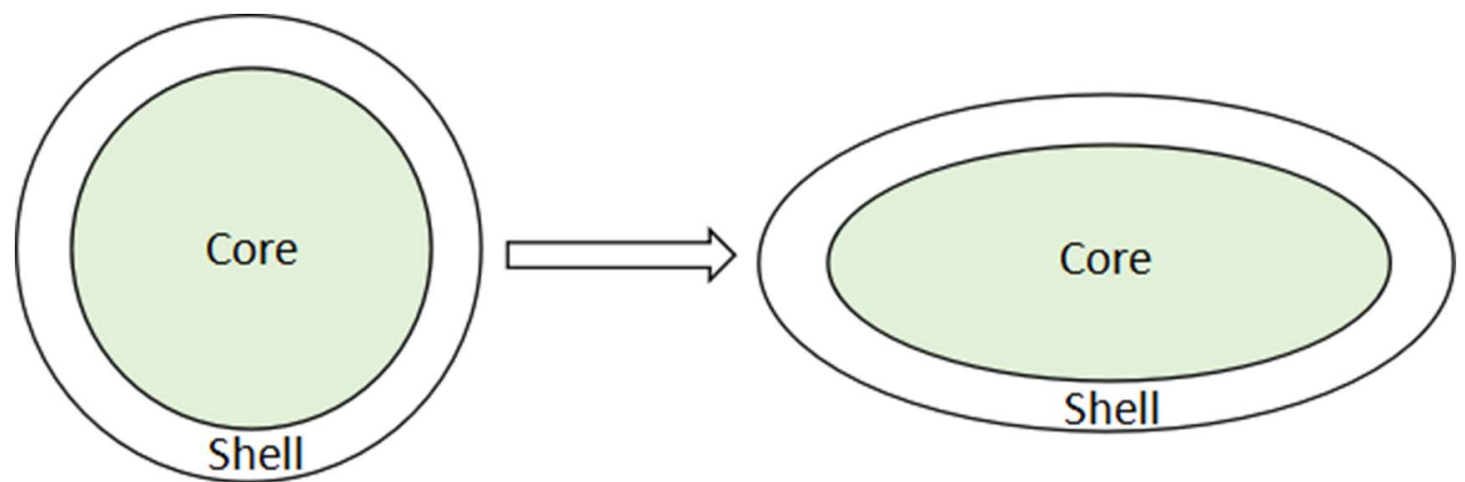

Figure 5. Changing the aspect ratio of the core-shell structure

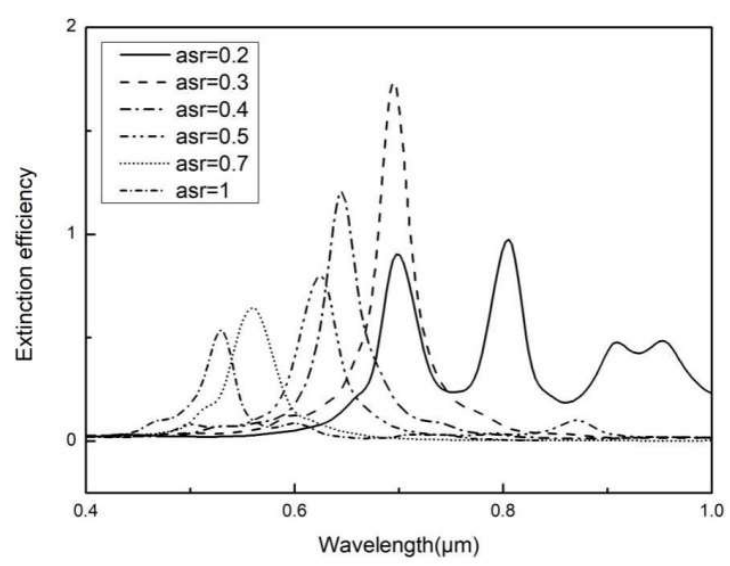

Figure 6. Extinction spectra for different aspect ratios of the core-shell structure

Figure 6 shows that the ratio of the minor axis to the major axis (the aspect ratio) played a very important role in optimizing the SPR peak as the ellipsoidal structure was constructed. We compared the extinction spectra of $\mathrm{Ag} @ \mathrm{TiO}_{2}$ CSNPs with six different aspect ratios, ranging from 0.2 to 1 . The thickness of the $\mathrm{TiO}_{2}$ shell layer was set at $1 \mathrm{~nm}$ while the Ag core radius was set at $26 \mathrm{~nm}$.

In Figure 6, as the aspect ratio increased from 0.2 to 1, the extinction efficiency increased and simultaneously the resonance frequency shifted to a lower value, showing a blue shift. Consequently, a spectrum with double resonance peaks was revealed, the first peak centered around $702 \mathrm{~nm}$ and the second around $810 \mathrm{~nm}$ for an aspect ratio of 0.2 . We believe that the appearance of these two plasmonic peaks was caused by structural anisotropic effects. When we further increased the aspect ratio, the amplitude of the SPR peak first increased, reaching a maximum aspect ratio of 0.3 , and then decreased as the aspect ratio was further increased. In summary, the fluctuations in the surface plasmon resonances with changes in the aspect ratios are caused by the size effect and the aspect ratio of shell layer to core.

\section{Conclusion}

In this paper, the DDA algorithm was used to simulate the extinction properties of composite nanoparticles with a core-shell structure and proved to be of great significance for the production and preparation of controlled coreshell composite nanoparticles with extinction peaks. Based on the DDA method, we found that the extinction efficiency for $\mathrm{Ag} @ \mathrm{TiO}_{2}$ was higher than for other CNNPs $\left(\mathrm{Au} @ \mathrm{SiO}_{2}, \mathrm{Ag} @ \mathrm{SiO}_{2}, \mathrm{Ag} @ \mathrm{TiO}_{2}, \mathrm{Au} @ \mathrm{Ag}\right.$, and $\left.\mathrm{Ag} @ \mathrm{Au}\right)$. The amplitude of the extinction spectrum decreased with increased shell thickness, and the wavelength of the extinction peak increased with increased core layer thickness. In addition, these simulation results showed that the aspect ratio played an important role in modulating the centered wavelength of surface plasmon resonance in the case of a spheroidal nanostructure. The extinction efficiency increased as the aspect ratio decreased, and the extinction peaks underwent a red-shift effect. When we set the aspect ratio to 0.3 , we obtained an optimal extinction result. All of 
these experiments prove that core-shell CNNPs with nanospheroid geometry are indeed a viable path for broadband tailoring of surface plasmon resonance, and we expect this to be a promising way of realizing a high-sensitivity SPR biosensor for applications in a variety of biomolecular reactions.

Author Contributions: Data curation, Wei Chen; Formal analysis, Chean-Cheng Su; Methodology, Jing Liu; Supervision, Chean-Cheng Su; Validation, Qiubo Ye and Cheng-Fu Yang; Writing - original draft, Wei Chen and Yushan Chen; Writing review and editing, Jing Liu and Yushan Chen.

Acknowledgments: This work was supported by the National Natural Science Foundation of China under Grant Nos. 61505068 and 11505079 and the Education and Scientific Research Project of the Fujian Provincial Education Department under Grant No. FBJG20170137.

Conflicts of Interest: The authors declare that there are no conflicts of interest regarding the publication of this paper.

\section{References}

1. Ghosh, B. K.; Ghosh, N. N. Applications of Metal Nanoparticles as Catalysts in Cleaning Dyes Containing Industrial Effluents: A Review. Journal of Nanoscience and Nanotechnology 2018, 18, pp. 3735-3758.

2. Dabrowski, M.; Lach, P.; Cieplak, M.; Kutner, W. Nanostructured molecularly imprinted polymers for protein chemosensing. Biosens. Bioelectron. 2018, 102, pp. 17-26.

3. Hsu, S. C.; Chuang, Y. C.; Sneed, B. T.; Cullen, D. A.; Chiu, T. W.; Kuo, C. H. Turning the Halide Switch in the Synthesis of Au-Pd Alloy and Core-Shell Nanoicosahedra with Terraced Shells: Performance in Electrochemical and Plasmon-Enhanced Catalysis. Nano Letters 2016, 16, pp. 5514-5520.

4. Boken, J.; Khurana, P.; Thatai, S.; Kumar, D.; Prasad, S. Plasmonic nanoparticles and their analytical applications: A review. Applied Spectroscopy Reviews 2017, 52, pp. 774-820.

5. Melinon, P.; Begin-Colin, S.; Duvail, J. L.; Gauffre, F.; Boime, N. H.; Ledoux, G.; Plain, J.; Reiss, P.; Silly, F.; Warot-Fonrose, B. Engineered inorganic core/shell nanoparticles. Physics Reports-Review Section of Physics Letters 2014, 543, pp. 163-197.

6. D'Addato, S.; Spadaro, M. C. Low pressure bottom-up synthesis of metal@oxide and oxide nanoparticles: control of structure and functional properties. Phys. Scr. 2018, 93, pp. 20.

7. Kang, F. W.; He, J. J.; Sun, T. Y.; Bao, Z. Y.; Wang, F.; Lei, D. Y. Plasmonic Dual-Enhancement and Precise Color Tuning of Gold Nanorod@SiO2 Coupled Core-Shell-Shell Upconversion Nanocrystals. Adv. Funct. Mater. 2017, 27, pp. 11.

8. Steer, D.; Kang, M.; Leal, C. Soft nanostructured films for directing the assembly of functional materials. Nanotechnology 2017, 28, pp. 17.

9. Shahamirifard, S. A.; Ghaedi, M.; Montazerozohori, M. Application of nanostructure ZnLI2 complex in construction of optical pH sensor. Applied Organometallic Chemistry 2018, 32, pp. 11.

10. Shah, N.; Claessyns, F.; Rimmer, S.; Arain, M. B.; Rehan, T.; Wazwaz, A.; Ahmad, M. W.; Ul-Islam, M. Effective Role of Magnetic Core-Shell Nanocomposites in Removing Organic and Inorganic Wastes from Water. Recent Pat. Nanotechnology 2016, 10, pp. 202-212.

11. Khalil, I.; Rahmati, S.; Julkapli, N. M.; Yehye, W. A. Graphene metal nanocomposites - Recent progress in electrochemical biosensing applications. J. Ind. Eng. Chem. 2018, 59, pp. 425-439.

12. Liu, S. Q.; Zhang, N.; Xu, Y. J. Core-Shell Structured Nanocomposites for Photocatalytic Selective Organic Transformations. Part. Part. Syst. Charact. 2014, 31, pp. 540-556.

13. Nayak, S.; Mahender, C.; Soam, A.; Nanda, J. Structural and optical studies of BiFeO3@SiO2 core/shell nanoparticles. Mater. Res. Express 2017, 4, pp. 7.

14. Wang, J. Q.; Shin, S. H. Room temperature nanojoining of Cu-Ag core-shell nanoparticles and nanowires'. J. Nanopart. Res. 2017, 19, pp. 13.

15. Das, S.; Paul, A.; Chattopadhyay, A. Nanocrystalline p-hydroxyacetanilide (paracetamol) and gold core-shell structure as a model drug deliverable organic-inorganic hybrid nanostructure. Nanoscale 2013, 5, pp. 9247-9254.

16. Das, S.; Chattopadhyay, A. Generation of inorganic-organic core-shell crystalline nanoparticles of silver and p-hydroxyacetanilide. RSC Adv. 2012, 2, pp. 10245-10250. 
17. Li, P.; Teng, Y. J.; Nie, Y. H.; Liu, W. H. SERS Detection of Insecticide Amitraz Residue in Milk Based on Au@Ag Core-Shell Nanoparticles. Food Anal. Meth. 2018, 11, pp. 69-76.

18. Saliminasab, M.; Garaei, M.; Moradian, R.; Nadgaran, H. Novel and Sensitive Core-Shell Nanoparticles Based on Surface Plasmon Resonance. Plasmonics 2018, 13, pp. 155-161.

19. Sharma, R.; Roopak, S.; Pathak, N. K.; Ji, A.; Sharma, R. P. Study of Surface Plasmon Resonances of Core-Shell Nanosphere: A Comparison between Numerical and Analytical Approach. Plasmonics 2017, 12, pp. 977-986.

20. Wang, Y.; Zhai, J.; Song, Y. L.; He, L. The Ag shell thickness effect of Au@Ag@SiO2 core-shell nanoparticles on the optoelectronic performance of dye sensitized solar cells. Chem. Commun. 2016, 52, pp. 2390-2393.

21. Hirakawa, T.; Kamat, P. V. Photoinduced electron storage and surface plasmon modulation in Ag@TiO2 clusters. Langmuir 2004, 20, pp. 5645-5647.

22. Pastoriza-Santos, I.; Koktysh, D. S.; Mamedov, A. A.; Giersig, M.; Kotov, N. A.; Liz-Marzan, L. M. One-pot synthesis of Ag@TiO2 core-shell nanoparticles and their layer-by-layer assembly. Langmuir 2000, 16, pp. 2731-2735.

23. El-Gohary, S. H.; Choi, M.; Kim, Y. L.; Byun, K. M. Dispersion Curve Engineering of TiO2/Silver Hybrid Substrates for Enhanced Surface Plasmon Resonance Detection. Sensors 2016, 16, pp.

24. Draine, B. T.; Flatau, P. J. DISCRETE-DIPOLE APPROXIMATION FOR SCATTERING CALCULATIONS. Journal of the Optical Society of America a-Optics Image Science and Vision 1994, 11, pp. 1491-1499.

25. Zhu, S.; Du, C.; Fu, Y. Localized surface plasmon resonance-based hybrid Au-Ag nanoparticles for detection of Staphylococcus aureus enterotoxin B. Optical Materials 2009, 31, pp. 1608-1613.

26. Sherry, L. J.; Chang, S. H.; Schatz, G. C.; Van Duyne, R. P.; Wiley, B. J.; Xia, Y. N. Localized surface plasmon resonance spectroscopy of single silver nanocubes. Nano Letters 2005, 5, pp. 2034-2038. 